\title{
The Method of Transfer Matrix and the Efficiency of the Sports Training
}

\author{
Youcai Xue \\ School of Science, Zhejiang University of Science and Technology \\ Hangzhou 310023, China \\ E-mail: xueyoucai@sohu.com
}

Received: November 4, 2010

Accepted: December 10, 2010

doi:10.5539/mas.v5n3p242

\begin{abstract}
The efficiency of sports training is an important parameter to judge the level of different coaches and their ability. To determine the training efficiency of the coaches, we should, firstly, remove the fundamental diversities of the different athletes and then judge them on the same level. Secondly, we should also pay more attention to the progress of the athlete, that is to say that our evaluation ought to focus on the variation made by the athletes who are directed by the coaches. This paper applies the method of one - step transfer probability matrix in the Markov Chains, and indicates the definition of the original state vector, the state transfer matrix, the state transfer degree matrix, the efficiency degree and so on. Then a mathematical model about the efficiency of the coach is established and the criteria steps are shown. The injustice of the evaluation by the fundamental diversities of different athletes is removed from the model, and the result reflects the degree of the improvement and transformation of the athlete, furthermore reflects the training efficiency and the training level of the coach accurately. At last an example and a piece of computer program to solve this problem are given.
\end{abstract}

Keywords: Sports training, Efficiency, Transfer matrix, Mathematical model, Criterion program

\section{Introduction}

The efficiency of sports training is a critical parameter to judge the level of the coach and his training. It's easy to find the efficiency of the coach's training if he can get a good achievement, but for other coaches, it will be difficult to quantify their training level. For example, because of the distinguish level of the different athletes, it is very difficult to determine the training level of the different coaches. How to set up a reasonable, quantitative method to judge the training level of the coaches is an urgent problem for the department of the management of the sports to solve.

To determine the efficiency and level of the training of the coach, firstly, we have to remove the fundamental distinguish of the different athlete. Then the coaches can be determined from the same foundation. Secondly, we should pay more attention to the improvement of the athletes, that is to say the judge should focus on the change of the athlete directed by the coach. For example, an original level 3 athlete was improved to level 2 or level 1 after a period of training, this is an improvement. Since there are so many factors to influence the training procedure, the factors affecting the consequence of the training are also plenty. To simplify the judge, we only have to get the state transformation of the athlete, therefore delete the fundamental distinguish and at the same time show the efficiency and level of the coach's training.

The article use the method called "one-step transfer probability matrix" in the Markov Chains and combine the method in articles (Wan-li Liu, Jie-qun Tan. 2002)(Guang-hai Li, Qun-fa Cui. 2008) to establish the definition of the state transition matrix of the athletes and the degree of the efficiency. Furthermore we set up a more suitable, practical mathematical model to determine the training efficiency of the coach and show the computer programming to realize it, which makes our model more general.

\section{Mathematical model}

\section{Fundamental definition}

Suppose there are $p$ coaches, $q$ athletes in one team. Firstly, we divide the athletes directed by different coaches into $m$ levels according to the achievement they have got when they joined the team, and compute the number of the athletes $n_{i}^{k}(i=1,2, \cdots, m, k=1,2, \cdots, p)$ of different levels directed by the $k$ th $(k=1,2, \cdots, p)$ coach, then we get the vector as following: 


$$
\alpha^{k}=\left(\begin{array}{llll}
n_{1}^{k} & n_{2}^{k} & \cdots & n_{m}^{k}
\end{array}\right), \quad k=1,2, \cdots, p
$$

We call it the original state vector.

To determine the training efficiency of the coach, we divide the behaviour of the athlete in a phase (in years or in months) into $m$ levels, too. This kind of division can be determined by combining with the training achievement of the usual time, phase competition. We denote the $i$ level athlete changed into $j$ levels as $n_{i j}^{k}(i, j=1,2, \cdots, m)$.

Definition 1 The matrix

$$
A^{k}=\left(\begin{array}{cccc}
n_{11}^{k} & n_{12}^{k} & \cdots & n_{1 m}^{k} \\
n_{21}^{k} & n_{22}^{k} & \cdots & n_{2 m}^{k} \\
\cdots & \cdots & \cdots & \cdots \\
n_{m 1}^{k} & n_{m 2}^{k} & \cdots & n_{m m}^{k}
\end{array}\right)=\left(n_{i j}^{k}\right)_{m \times m}, T^{k}=\left(\begin{array}{cccc}
\frac{n_{11}^{k}}{n_{1}^{k}} & \frac{n_{12}^{k}}{n_{1}^{k}} & \cdots & \frac{n_{1 m}^{k}}{n_{1}^{k}} \\
\frac{n_{21}^{k}}{n_{2}^{k}} & \frac{n_{22}^{k}}{n_{2}^{k}} & \cdots & \frac{n_{2 m}^{k}}{n_{2}^{k}} \\
\cdots & \cdots & \cdots & \cdots \\
\frac{n_{m 1}^{k}}{n_{m}^{k}} & \frac{n_{m 2}^{k}}{n_{m}^{k}} & \cdots & \frac{n_{m m}^{k}}{n_{m}^{k}}
\end{array}\right)=\left(t_{i j}^{k}\right)_{m \times m}
$$

are called the state transfer matrix and the state transfer degree matrix of the athlete. Obviously, $t_{i j}^{k} \geq 0, \quad \sum_{j=1}^{m} t_{i j}^{k}=1, i=1,2, \cdots, m$.

Definition 2 Matrix $S^{k}=\left(s_{i j}^{k}\right)_{m \times m}$ is the progressive matrix of the state transfer matrix. and the element $S^{k}$ of the progressive matrix

$$
s_{i j}^{k}=(i-j)^{3} t_{i j}^{k}=(i-j)^{3} \frac{n_{i j}^{k}}{n_{i}^{k}}, i, j=1,2, \cdots, m, k=1,2, \cdots, p
$$

is the progress degree of $t_{i j}^{k},(i-j)^{3}$ is the weight of the transfer degree $t_{i j}^{k}$.

Here, in order to show the rewards and punishment principal, we let the weight of transfer degree $t_{i j}^{k}$ be $(i-j)^{3}$. For example, if an athlete has changed from $i$ th level into $j$ th level, such as from level 3 to level 1 , and then the weight is $(3-1)^{3}=2^{3}=8$. But if he has changed from level 1 to level 2 , the weight is $(1-2)^{3}=(-1)^{3}=-1$. If the athlete makes the progress, the weight is positive, otherwise, the weight is negative. Then the reward and punishment are obvious. We take the parameter of the weight 3 ; one reason is to show the reward and punishment, the more progress and regress, the more reward and punishment. The other reason is we can distinguish the positive weight value from the negative value easily. If the weight value of 3 is too large, we can take value 2. Meanwhile, to indicate the positive and negative calculation of the weight, we can evaluate the value of progress degree $s_{i j}^{k}$ as following:

$$
s_{i j}^{k}= \pm(i-j)^{2} t_{i j}^{k}= \pm(i-j)^{2} \frac{n_{i j}^{k}}{n_{i}^{k}}, i, j=1,2, \cdots, m, j=1,2, \cdots, p
$$

We take the positive or negative value according to the progress or regress. In principal, the parameter of the weight can be any real number greater than 1 . For example, the value can be 2.5 etc.

Furthermore, we can use the following method to determine the weight of the transfer degree

$$
s_{i j}^{k}=\left\{\begin{array}{ll}
-(i-j)^{2} t_{i j}^{k}=-(i-j)^{2} \frac{n_{i j}^{k}}{n_{i}^{k}}, & i \leq j \\
{\left[(i-j)^{2}+(i-j)\right] t_{i j}^{k}=\left[(i-j)^{2}+(i-j)\right] \frac{n_{i j}^{k}}{n_{i}^{k}},} & i>j
\end{array} \quad i, j=1,2, \cdots, m\right.
$$


The weight in (4) is to improve the degree of the rewards, and of course we can let the weight value be 3 , too. When we think about the progress degree $s_{i j}^{k}$, we can also put the coefficient $\beta$, the difficulties of the competition, into the formula. For example, the high level competition has a greater coefficient than the low level competition, and then we can evaluate the progress degree $s_{i j}^{k}$ as following:

$$
\begin{aligned}
& s_{i j}^{k}=(i-j)^{3} \beta t_{i j}^{k}=(i-j)^{3} \beta \frac{n_{i j}^{k}}{n_{i}^{k}}, i, j=1,2, \cdots, m \\
& s_{i j}^{k}= \pm \beta(i-j)^{2} t_{i j}^{k}= \pm \beta(i-j)^{2} \frac{n_{i j}^{k}}{n_{i}^{k}}, i, j=1,2, \cdots, m \\
& s_{i j}^{k}=\left\{\begin{array}{l}
-(i-j)^{2} \beta t_{i j}^{k}=-(i-j)^{2} \beta \frac{n_{i j}^{k}}{n_{i}^{k}}, i \leq j \\
{\left[(i-j)^{2}+(i-j)\right] \beta t_{i j}^{k}=\left[(i-j)^{2}+(i-j)\right] \beta \frac{n_{i j}^{k}}{n_{i}^{k}}, \quad i>j}
\end{array} \quad i, j=1,2, \cdots, m\right.
\end{aligned}
$$

in these formulae, when they are in the same level competition, the coefficient $\beta=1$; when they are in a higher level competition, the coefficient $\beta>1$, maybe $\beta=2$; when one of a higher level competes with the one of the lower level, the coefficient $0<\beta<1$.

2.2 Mathematical model and method of evaluation

Definition $3 \mathrm{We}$ call

$$
E\left(S^{k}\right)=(1,1, \cdots, 1)\left(\begin{array}{cccc}
s_{11}^{k} & s_{12}^{k} & \cdots & s_{1 m}^{k} \\
s_{21}^{k} & s_{22}^{k} & \cdots & s_{2 m}^{k} \\
\cdots & \cdots & \cdots & \cdots \\
s_{m 1}^{k} & s_{m 2}^{k} & \cdots & s_{m m}^{k}
\end{array}\right)\left(\begin{array}{c}
1 \\
1 \\
\cdots \\
1
\end{array}\right)=\sum_{i, j=1}^{m} s_{i j}^{k}
$$

the efficiency degree of the coach.

By the definition of the efficiency degree of the coach, we can take the comparison and evaluation. The steps are shown as Following:

(1) According to the basic condition of the athletes when they joined the team, we divide the athletes into $m$ levels, and subdivide the athletes of different levels into $m$ levels, based on the achievement of different period of time.

(2) Evaluate the state transfer matrix $T^{k}$.

(3) According to the practical circumstances, determine the weight parameter of the transfer progress degree and the coefficient of the difficulty, choose one formula from (2-7) to computer the transfer progress, and find the transfer matrix $S^{k}$.

(4) Investigate the efficiency degree of the coaches by formula (8).

(5) By comparing the value of $E\left(S^{k}\right)$, we can get the training consequence of the different coaches easily.

\section{The computer programming of the efficiency degree computing.}

In order to make our model more convenient and more practical, we design the computer program by Matlab. In practical, people just input the correspondence original state vector and choose the computing formula from (2-7) to evaluate the progress degree $s_{i j}^{k}$, they can get the consequence directly. (When choosing formula $5,6,7$, the difficulty coefficient $\beta$ should be given.)

Example 1. We want to compare the training efficiency of three coaches, we denote the number of the athletes they direct as $\alpha^{1}=\left(\begin{array}{lll}3 & 4 & 2\end{array}\right), \alpha^{2}=\left(\begin{array}{lll}2 & 4 & 3\end{array}\right), \alpha^{3}=\left(\begin{array}{lll}3 & 3 & 2\end{array}\right)$, and after a period time of training, the level of the athletes changed into: 


$$
A^{1}=\left(\begin{array}{lll}
2 & 1 & 0 \\
1 & 2 & 1 \\
0 & 1 & 1
\end{array}\right), A^{2}=\left(\begin{array}{lll}
1 & 1 & 0 \\
0 & 2 & 2 \\
0 & 1 & 2
\end{array}\right), A^{3}=\left(\begin{array}{lll}
1 & 2 & 0 \\
0 & 2 & 1 \\
0 & 1 & 1
\end{array}\right)
$$

We can use our method to compare the three coaches.

The Matlab program is shown as following:

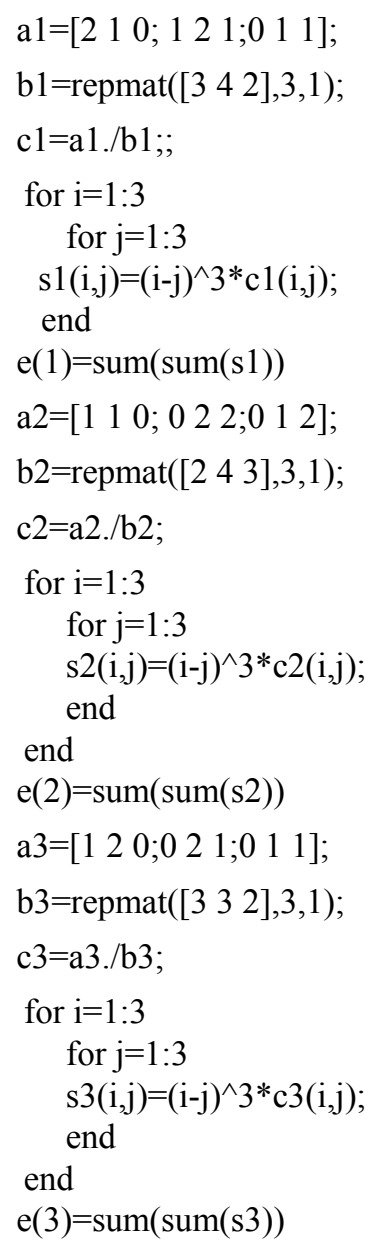

The output is

ans $=$

$\begin{array}{lll}-0.1667 & -0.6667 & -0.8333\end{array}$

Since $E\left(S^{1}\right)$ is the greatest efficiency degree, our consequence is that the first coach has the best training efficiency.

\section{References}

Guang-hai Li, Qun-fa Cui. (2008). Application of Markov Normal Process to Evaluation in Education Quality. Journal of Anyang Teachers College, 2008(2), 26-28.

Wan-li Liu, Jie-qun Tan. (2002). Application of Transfer matrix analysis to the Teaching Effect, System Engineering- Theory \& Practical. 2002(6), 135-138.

You-cai Xue, Shi-ping Ruan. (2009). The matrix model for sports club to introduce elite athletes. Linear Algebra. 2009, 55-58.

Zhou Sheng. Probability and Statistics. (3rd version). Higher Education Press, 355-362. 